\title{
REAL FLIGHT DEMONSTRATION OF PITCH AND ROLL CONTROL FOR UAV CANYON FLIGHTS
}

\author{
Cezary KOWNACKI* \\ *Faculty of Mechanical Engineering, Department of Automatics and Robotics, Bialystok University of Technology, \\ ul. Wiejska 45C, 15-351 Bialystok, Poland \\ c.kownacki@pb.edu.pl
}

\begin{abstract}
The paper presents results of an experiment prepared to validate the autonomous control of obstacle avoidance designed for a micro UAV to fly in urban canyons. The idea of the obstacle avoidance assumes usage of two miniature laser rangefinders responsible for obstacle detection and range measurement. Measured ranges from obstacles placed on both sides of UAV can be used to simultaneous control of desired roll and pitch angles. Such combination of controls allows achieving high agility of UAV, because during a maneuver of obstacle avoidance UAV can make a turn and climb at the same time. In the experiment, controls of roll and pitch angles were verified separately to ensure high reliability of results and clearance of UAV behavior in the real flight. Because of lack of appropriate objects, which can be used as obstacles, laser rangefinders were directed vertically to the ground instead of the original horizontal configuration. So sensors determine ranges from the ground during a descent flight of UAV, and if their values are lower than defined threshold, it could be interpreted as obstacle detection. The experiment results present UAV behavior adequate to designed controls of roll and pitch angle. The vehicle turns in the opposite direction to the sensing axis of laser rangefinder detecting an obstacle and starts climbing when both sensors detect obstacles at the same range below the threshold.
\end{abstract}

Key words: Obstacle Avoidance, Autonomous Control, Unmanned Air Vehicle, Reactive Control, Urban Canyon

\section{INTRODUCTION}

Unmanned aerial vehicles have become increasingly popular within the last few years. They are applicable successfully in many different areas of civil engineering and research fields where fast, reliable and low cost inspection is expected (Campoy et al., 2009). Most common use of micro and mini UAVs is undertaking patrol flights for police, fire-brigades and foresters. Using UAVs allows observing with much wider field of vision than it is possible on the ground. Even so UAV is still associated mostly with military applications such as intelligence where only larger vehicle can be used. To change this meaning, micro and mini UAV must become more autonomous and intelligent to be able to operate in zone nearby humans being, i.e. in urban environments. It would be possible if UAV was able to fly autonomously and safely for surrounding objects ensuring the lowest risk of collision. So the primary aim of autonomous UAV is being able to see and avoid any obstacle. Today technology still seems to be limiting possibilities to solve this problem entirely for micro UAV. In spite of that many researchers are still attempting to demonstrate autonomous UAV having at least partial possibilities of obstacle avoidance (Beyeler et al., 2009; Griffiths et al., 2007]. Such case of partial obstacle avoidance is an autonomous flight in unknown canyons (Kownacki, 2009; Kownacki, 2010; Kownacki, 2011; Hrabar et al., 2006). It assumes that UAV flies between obstacles like buildings or canyon walls. Therefore, it requires continuous determining ranges from two obstacles on both sides of the canyon. Then the main task of control system is to place flying vehicle in the center of canyon. Ranges from canyon walls can be determined by Fig. 1):

- a pair of laser rangefinders (Kownacki, 2009; 2010; 2011),

- optical flow sensors or miniature cameras (Beyeler et al.,
2009; Griffiths et al., 2007; Hrabar et al., 2006; 2009),

- a single camera and optical flow processing (Andert et al., 2010).
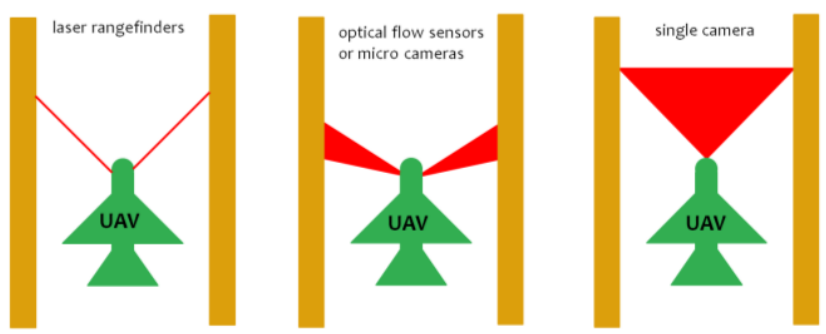

Fig. 1. Range measurement applied in realization of the flight in a canyon

In (Hrabar et al., 2009) authors presented results of vehicle flights in urban canyons using two miniature cameras and optical flow image processing. The results were impressive, but in this case the authors used a vertical taking off and landing vehicle, which are able to hover or to fly extremely slowly. So it was much easier than realizing an autonomous flight in a canyon with a fixed wing vehicle. Therefore, more attractive results, there are in (Griffiths et al., 2007) where authors used a delta wing vehicle equipped with a laser rangefinder applied to the frontal obstacle avoidance, and two optical flow sensor applied to the flight in a canyon. The idea of vehicle control is similar as in the previous work, but this time the vehicle flies much faster. Both works use the range measurement derived from optical flow technique. Similarly to the others optical measurements, it also remains sensitive to exposure parameters like a light intensity and diversity of the image frame. Hence, we aim to realize the control of UAV flight across the canyon in a little bit different way. Instead 
of optical flow sensors or cameras we employed two tiny laser rangefinders (MLR100) (Kownacki, 2009; 2010; 2011). These robust sensors determine ranges from obstacles at the vehicle front on both sides of the canyon (Fig. 2) (Kownacki, 2009; 2010; 2011). The effective sensing range is about 150 meters without disturbances. The limitation of proposed method is that it is blind to narrow frontal obstacles. It can be accepted since the method was designed especially for canyon flights, where the obstacles are to the sides. So it is well suited to autonomous urban flights and this is the main aim of the research.

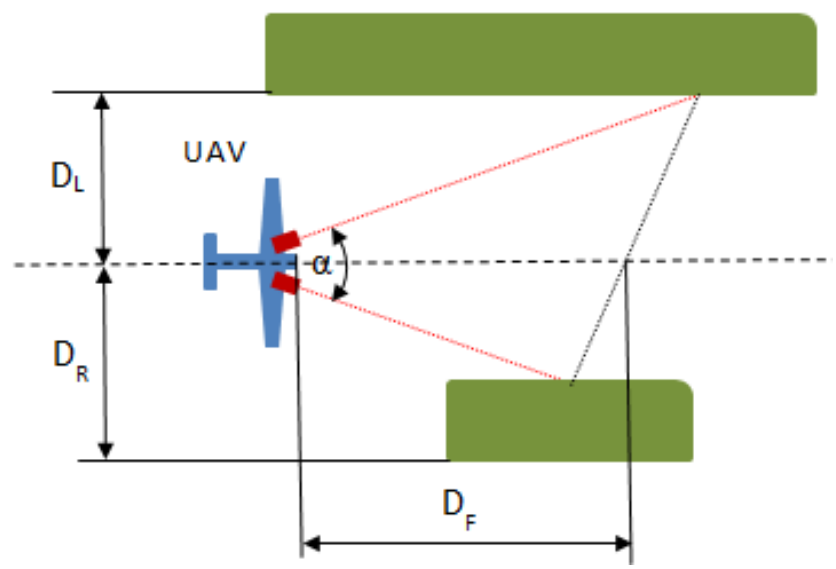

Fig. 2. The idea of flight in a canyon based on two laser rangefinders

The whole idea of the proposed concept of autonomous obstacle avoidance and flight in urban canyons was already fully described in the author's pervious works (Kownacki, 2009; 2010; 2011). Results derived from flight simulations present perfect possibilities of the concept, and it state, that it can be implemented in a real micro UAV easily. Now we would like to show results from the experiment using a real micro UAV, which verifies usefulness of the concept.

\section{THE CONTROL OF ROLL AND PITCH ANGLES}

According to the concept presented in (Kownacki, 2011), ranges from obstacles acquired by two laser rangefinders can be used as inputs of the roll angle control. The roll angle control splits into two levels. The high level contains two PID loops responsible for determining desired roll angles corresponding to the appropriate sensor. Range from the left sensor expressed in y axis of the vehicle body frame is the input of the first PID loop producing positive desired roll angle, what will make UAV turning right. Similarly, range from the right sensor expressed in y axis of the vehicle body frame is the input of the second loop with negative desired roll angle on the output and this time it will make UAV turning left. Of course, the resultant desired roll angle is a sum of both PID loops' outputs. The low level of roll angle control keeps the error between actual and desired angle nearby zero, and it is a part of original autopilot firmware. If both PIDs' outputs are zero, the low level roll control will acquire desired roll angle from navigation task. The avoidance controller toggles the source of desired roll angle (Kownacki, 2011). The high and low level of roll angle control required a modification of original autopilot firmware code, what was possible using software delivered by autopilot manufacturer. Figure 3 presents the high of control of roll angle designed for autonomous flight in urban canyon. Work (Kownacki, 2011) present in details all necessary calculations applied in two blocks called respectively: frame translation and filtration, and avoidance controller. The first block is responsible for disturbances filtration and translation of measured ranges from body frame to the north east down frame. In this way, we eliminate roll and pitch angle impact on obstacle relative position. In turn, the second block i.e. avoidance controller is responsible for switching the roll control from navigation mode to obstacle avoidance mode (Kownacki, 2011).

The control of roll angle is responsible for turns, and it operates in both cases: obstacle avoidance and flight in urban canyon. What will happen, when frontal obstacle is appearing, and both sensors are measuring the same range? If the PIDs have the same output values only with opposite signs, the resultant desired roll angle will be equal zero and the vehicle will not turn. To solve this problem, we extend the obstacle avoidance control described in works (Kownacki, 2009; 2010) with the control of desired pitch angle (Kownacki, 2011).

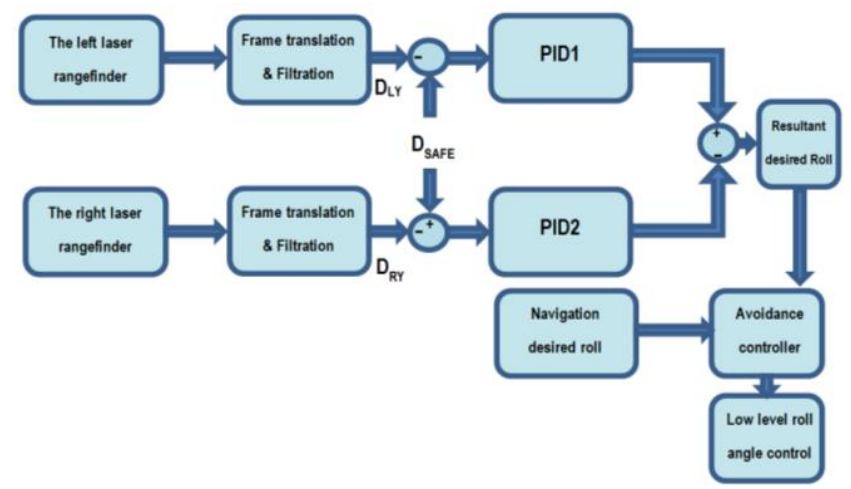

Fig. 3. The structure of high level of designed roll angle control

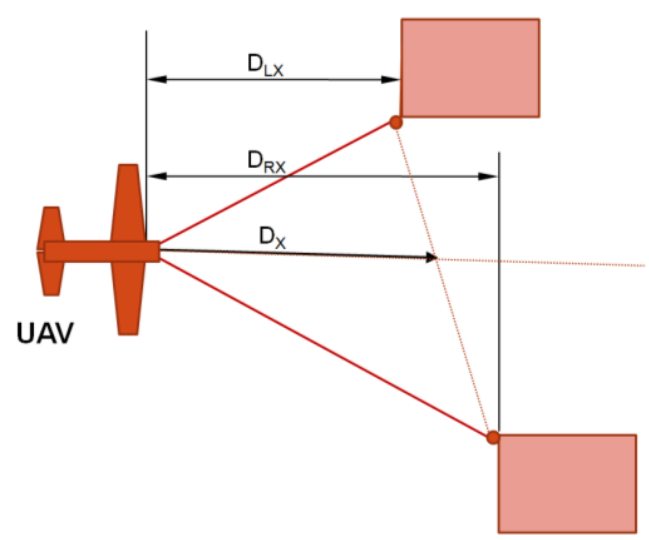

Fig. 4. The averaged range $D x$ from obstacles expressed in $x$ axis of UAV body frame

The control of desired pitch angle uses the averaged value of ranges from obstacles expressed in $\mathrm{x}$ axis of the vehicle body frame (Fig. 4). It also splits into two levels. The high level contains one PID loop, which generates a positive desire pitch angle, when the average range value in $x$ axis droops below the threshold value. Hence UAV is able to climb without making a turn. The roll angle control and pitch control angle operates simultaneously, so it is possible to combine turning with climbing. This is a significant advantage of the proposed obstacle avoidance control. The averaged range in $x$ axis is the input of the PID loop. The low level control of pitch angle is responsible for realizing desired pitch 
angle derived from the PID or navigation task of autopilot. The avoidance controller also toggles the source of desired pitch.

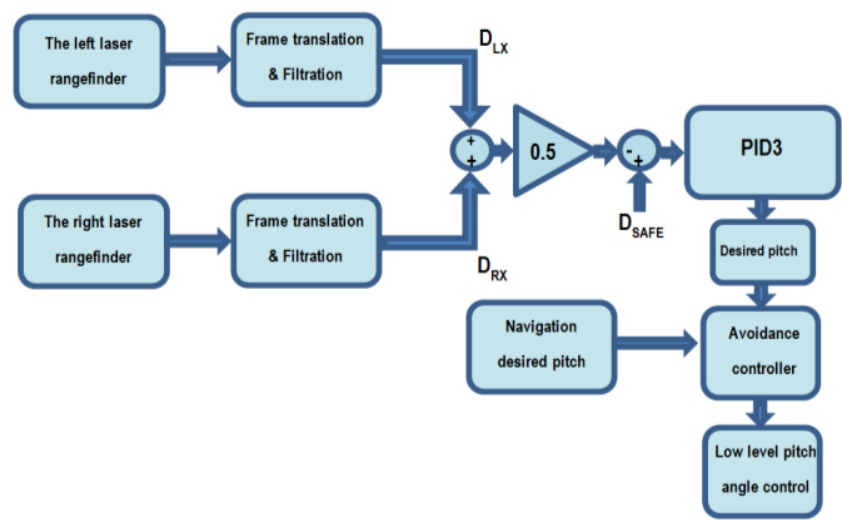

Fig. 5. The structure of high level of designed pitch angle control

Because both designed roll and pitch controls are inputs for the low level control loops, it secures aircraft against unstability in the case of severe roll or pitch command.

Fig. 5 presents the diagram explaining the structure of the high level of desired pitch control.

\section{REAL FLIGHT EXPERIMENT}

A real flight experiments verify both controls of pitch and roll angles. The serious problem of scenario setting can be met in experiments respecting obstacle avoidance with fixed wing UAV. Nobody wants to crash vehicle during the first flight or to make any damage in third party objects. Fixed wing UAV flies safely at minimum altitude about 50 meters. It ensures that the vehicle will not be under the influence of turbulence created by lifting wind from the warm ground. Hence obstacles should have with 50 meters height at least. Because of lack of such objects satisfying safety conditions, we decided to test pitch and roll controls with distinctive sensors assembly. Measurement axes of both sensors are directed vertically to the ground level instead of the original horizontal placement from figure 2. Measurement axes were turned outside slightly about $20^{\circ}$ (Fig. 6) to decrease accidental reflections of laser beams from the vehicle body.

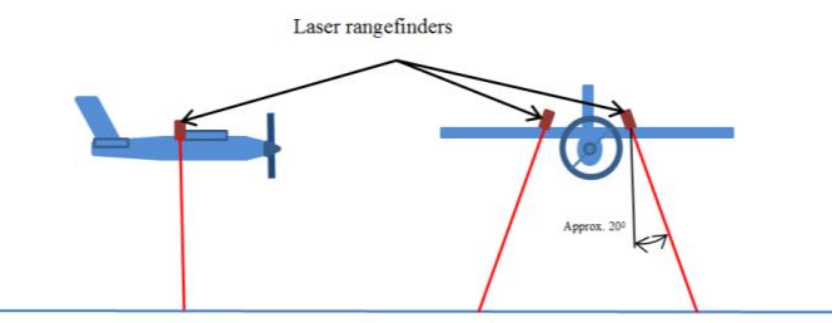

horizontal plane

Fig. 6. The laser rangefinders assembly applied in the experiment

The sensors assembly allows applying the ground level as an obstacle, when UAV will be decreasing its altitude. Because the maximum measurement range is about 150 meters, UAV can fly safely at altitudes higher than 50 meters during the experiment.
Of course it will not be the case with tall obstacles such as trees, but it will be well suited to urban canyon flights above trees-tops level. Fig. 7 presents the principle of the experiment. The experiment splits into two parts. The first part verifies the roll control, and we test the pitch control in the second.

During both parts of the experiment, UAV receives a command from GCS (Ground Control Station) to lower its altitude. It causes decrement of measured range, and this is synonymous with obstacle detection. In the first part of the experiment we disabled the left laser rangefinder, so the UAV behavior should be the same as in the case of obstacle detection on the vehicle right. UAV should start turning left when measured range droops below the threshold value Dsafe.

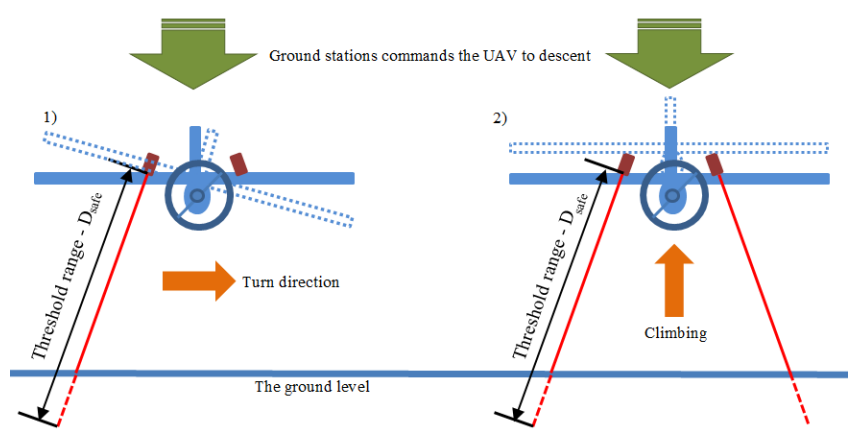

Fig. 7. The verification of roll and pitch controls designed for obstacle avoidance and flight in urban canyon. Orange arrows present expected response of UAV control to actual range measurement: 1) the roll control part of the experiment, 2) the pitch control part of the experiment

In the second part of the experiment, both sensors are left enabled. Then only the desired pitch angle derived from the high level of pitch control should be different from zero. The resultant desired roll angle should be zero, because the left and the right sensor measure the same range. It results only in UAV climbing, what overrides the command from GCS.

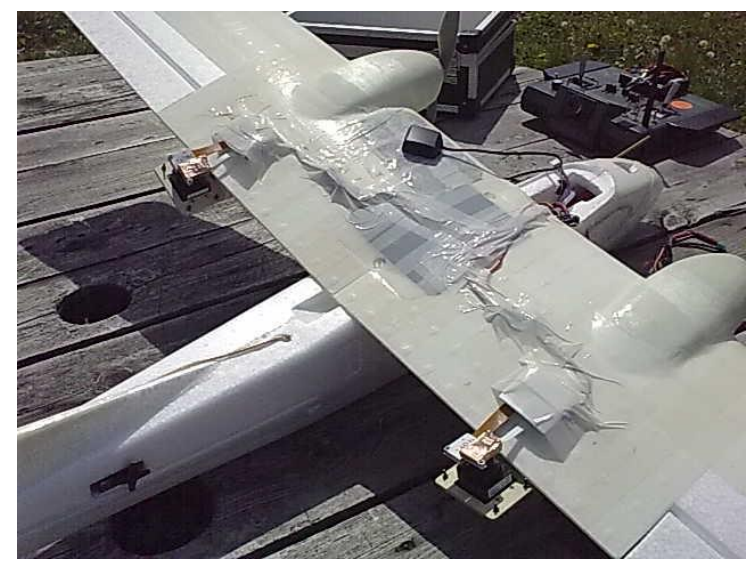

Fig. 8. Test UAV with two laser rangefinders fixed to its wings

\section{RESULTS}

Fig. 8 presents the flight path recorded during the first part of the experiment, i.e. verification of the roll control. We used four waypoints to create requested flight path to be realized 
by autopilot navigation task.

Fig. 9 clearly presents a turn associated with obstacle avoidance (marked dashed line in yellow color) produced by the designed roll control, while the vehicle is flying below 79 meters, the altitude related with threshold Dsafe $=85$ meters $\left(79 / \cos 20^{\circ}\right)$ (Fig. 6 and 10). The desired altitude set up by GCS command is equal 60 meters (at 390 second) and next 45 meters (at 410 second) (Fig. 10). When the vehicle returns to fly at an altitude higher than 79 meters, the requested flight path becomes valid again (orange dashed line in Fig. 9). Next Figures present flight parameters stored in the data log of autopilot. They describe the roll control operation and the vehicle behavior in details.

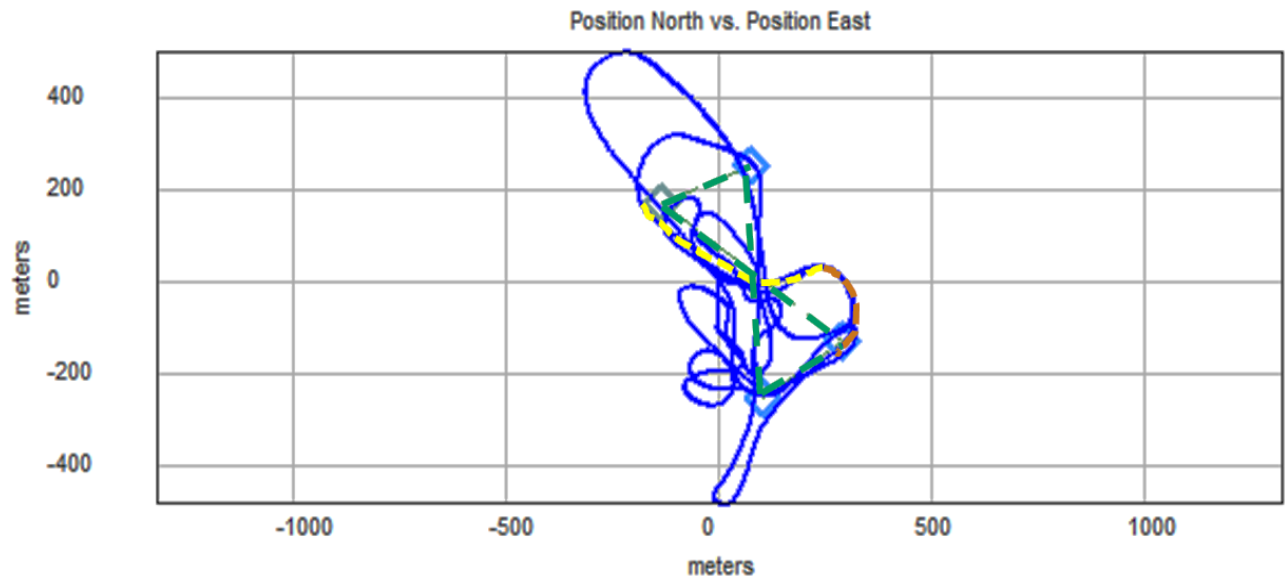

Fig. 9. The flight path recorded during verification of the roll control. The green dashed line represents the requested flight path to be realized by the autopilot navigation task. The yellow dashed line represents turn corresponding to obstacle avoidance, while UAV is flying at an altitude below 79 meters (related with Dsafe $=85$ meters). The orange dashed line represents a return to realize the requested flight path after climbing above the altitude of 79 meters
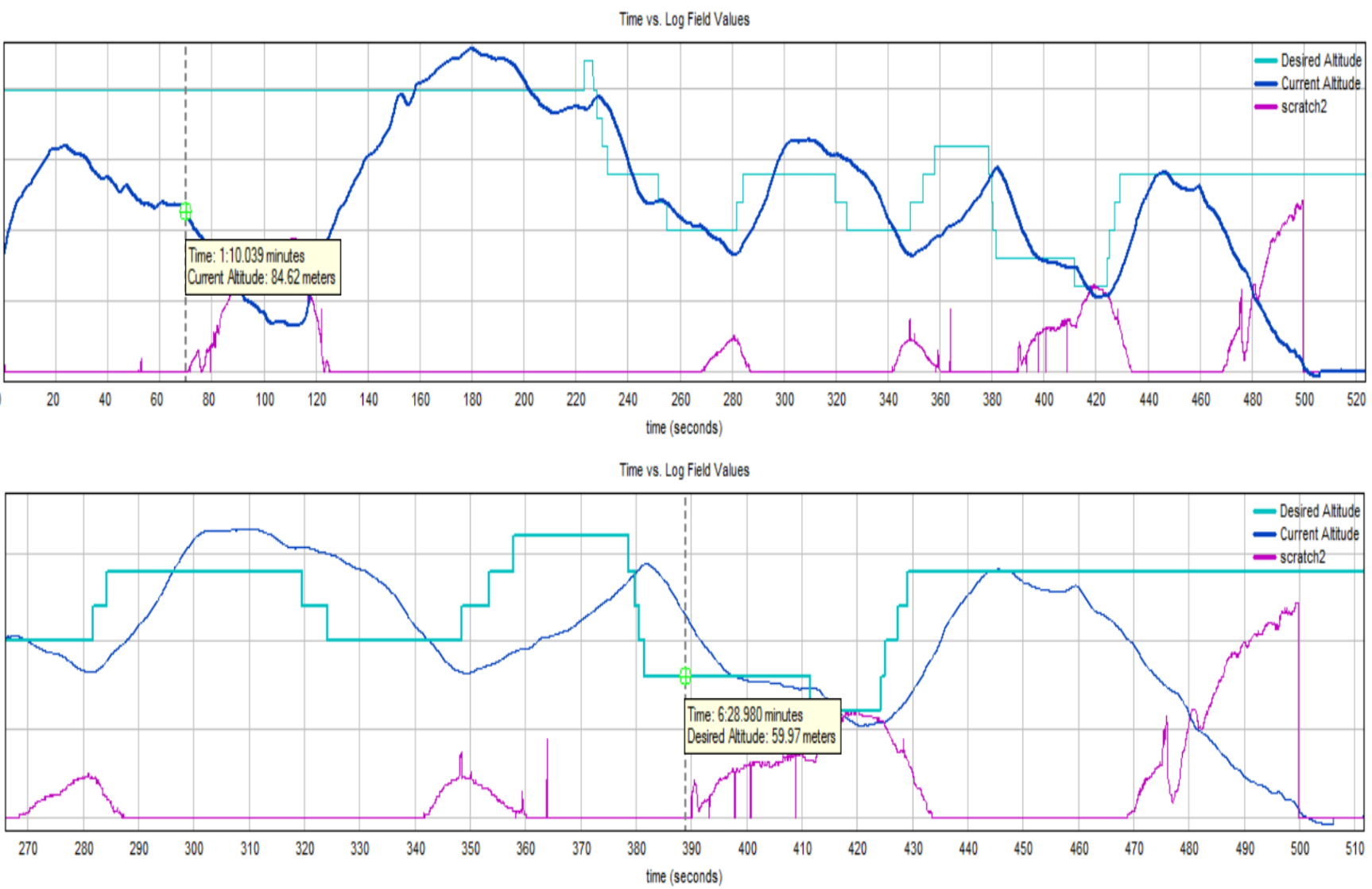

Fig. 10. The flight parameters recorded during the roll control verification. The current altitude plot is navy blue, the desired altitude plot is blue and the plot of current error value (scratch2) of PID associated with the right sensor is violet 


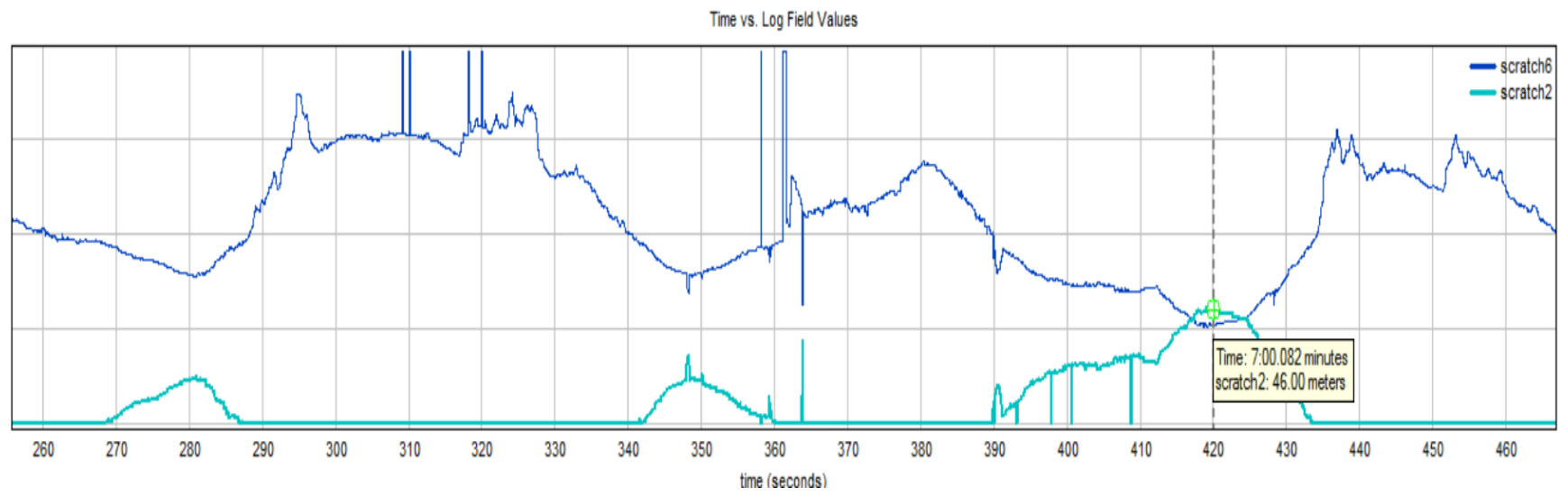

Fig. 11. Plots of scratch2 and scratch6 are respectively the current error value of PID associated with the right sensor and the range measured by the right sensor

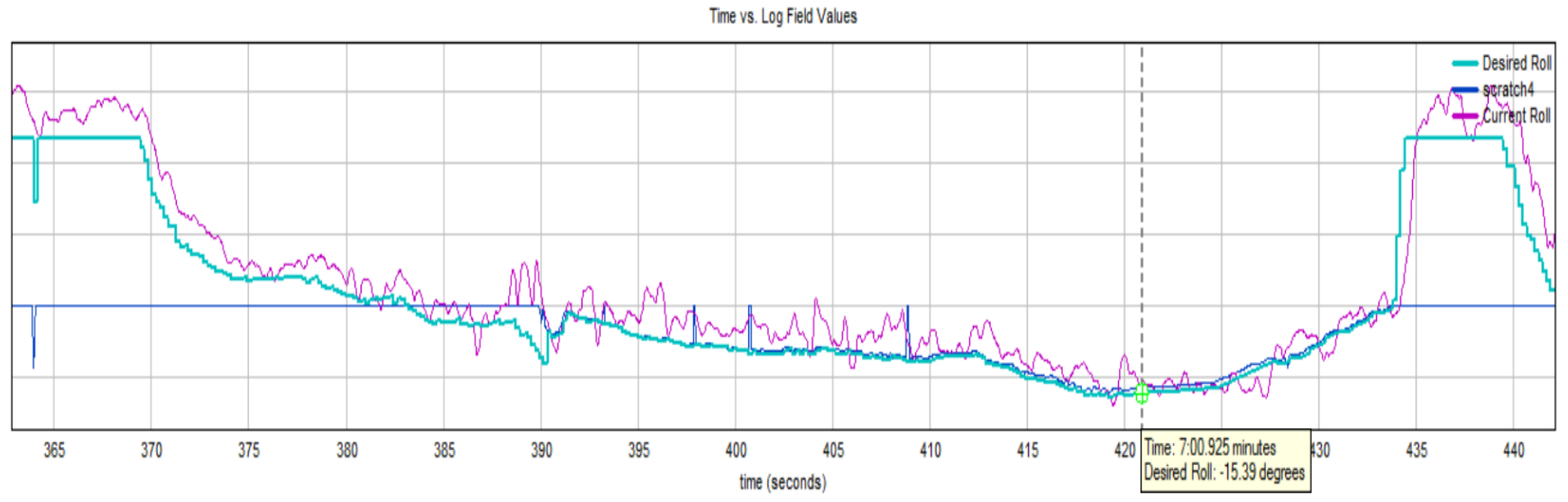

Fig. 12. Plots of the desired roll angle, the current roll angle and scratch4 - the current output value of PID associated with the right sensor.

The current PID output value is the same as the resultant desired roll angle, i.e. the output of the high level of roll control

In Fig. 10 we can see, that the current error value of the PID is greater than zero, while the current altitude is lower than 79 meters. The error reaches a maximum value 47 meters at the current altitude being equal 40 meters, while the measured range is 38 meters (419 second of the flight) (Fig. 11). The turn presented in Fig. 9 takes place between 390 and 433 second of the experiment flight. So we can notice in Fig. 12, that the output of PID associated with the right sensor is different from zero in the time range between 390 and 433 second of the flight. In the same range of time, the desired roll angle is equal the PID output. After the 430 second of the flight, UAV climbs above 79 meters (the desired altitude is 105 meters), and the low level of roll control is switched to navigation task. UAV returns to realize the requested flight path.

Fig. 13 presents the flight path recorded during the second part of the experiment. A red line represents the part of flight path where the pitch control was tested. The vehicle was flying over flat terrain.

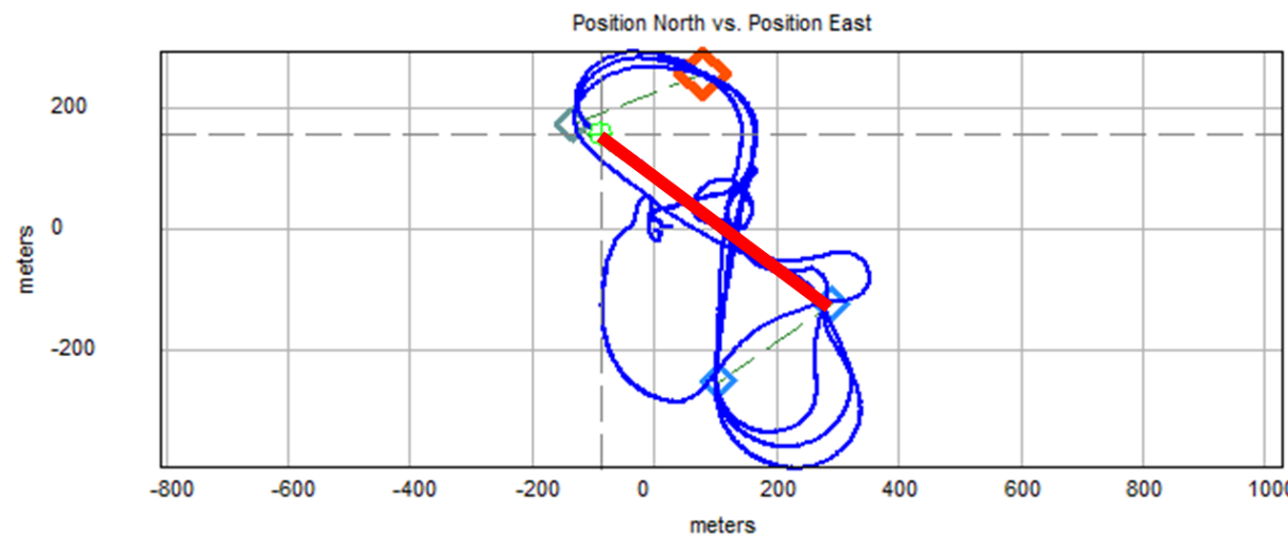

Fig. 13. The flight path recorded during verification of the pitch control 


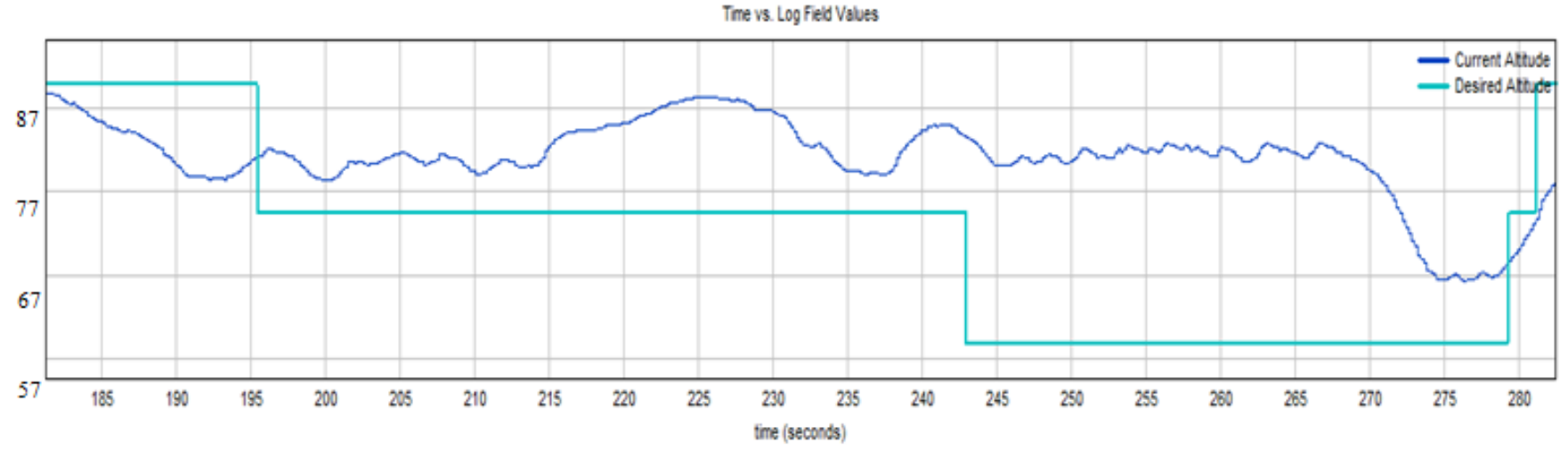

Fig. 14. The upper graph is a plot of current altitude and the bottom graph is a plot of desired altitude.

The current altitude never decreased below 80 meters even the desired altitude was 60 meters (at 243 second of the flight)

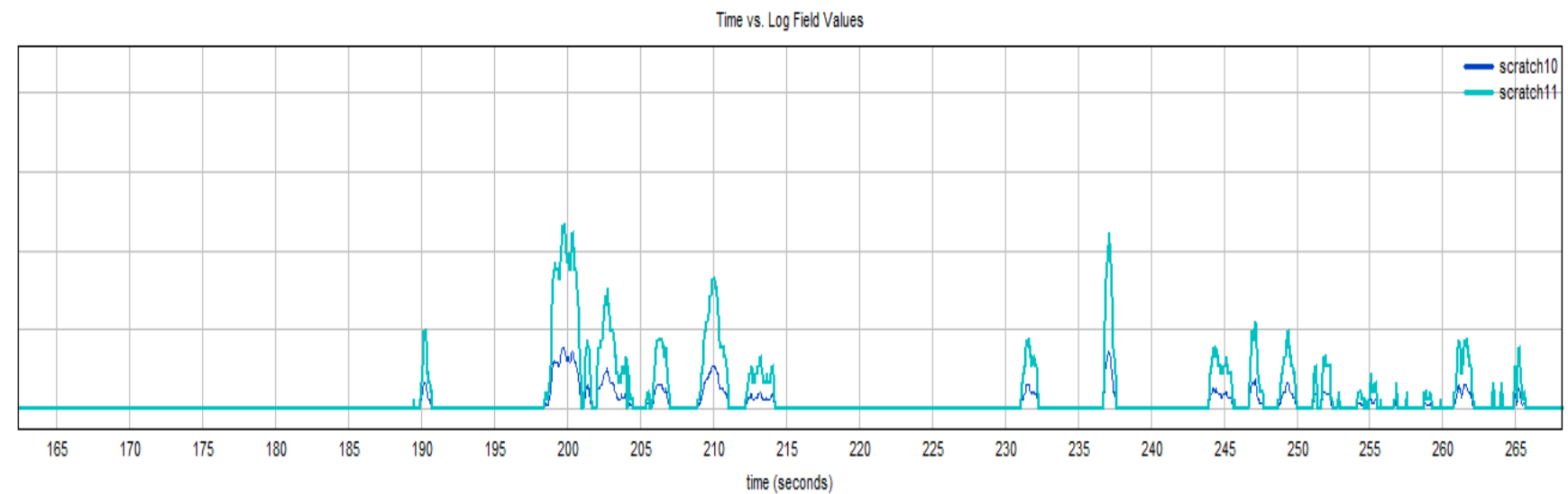

Fig. 15. Plots of scratch 10 and scratch 11 represents respectively the current error and the current output of the PID from the high level of pitch control

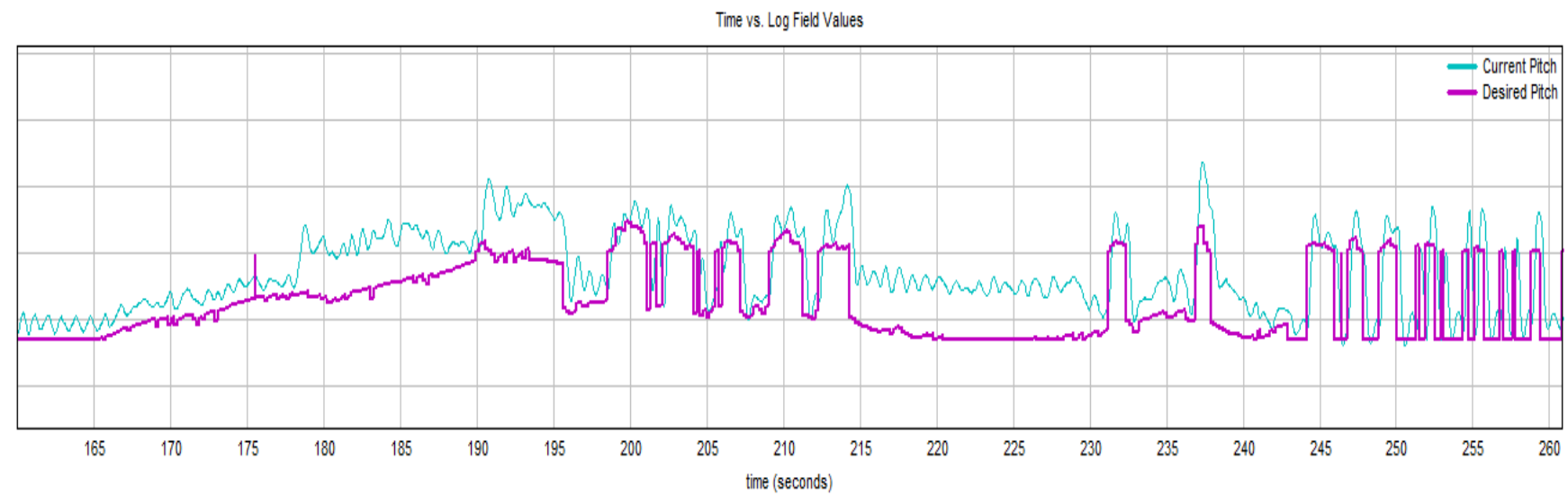

Fig. 16. Plots of current pitch and desired pitch related with Fig. 15

This time also the change of flight altitude simulates the obstacle detection. Fig. 14 presents the plot of current altitude and the plot of desired altitude recorded during the second part of the experiment.

It can be clearly underscore that the current altitude never decreased below 80 meters, while the desire altitude was decreasing from 90 to 60 meters with 15 meters steps. The current altitude oscillates around approx. 81 meters, what means that, the pitch control is being switched from obstacle avoidance to navigation task periodically. The altitude level equals 81 meters defines the moment of switching. Fig.15 presents plots of scratch 10 and scratch 11 , which are standing respectively for the current error and the current output of the PID from the high level of pitch con- trol. It is obvious that these plots correlate directly with the oscillation of current altitude.

The second part of experiment presents reliable view of UAV behavior and the pitch control operation. We can see that, in spite of command of desired altitude decreasing, UAV is not able to fly below specified level. This is a strong proof of the pitch control effectiveness, because the test flight can be easily compared with UAV level flight over a hill. In the test, we decreased desired altitude and UAV was not able to descent, what corresponds to UAV climbing as a consequence of measured range decreasing while desired altitude is constant. 


\section{CONCLUSIONS}

Results of the both parts of the experiments present that the roll and pitch controls operation meets fully and unquestionably all assumptions made during the design stage of proposed obstacle avoidance. UAV behavior was exactly the same as it should be in real obstacle avoidance. If the one laser rangefinder detects the range from obstacle below the safe threshold, UAV will make a turn in the opposite direction to the obstacle location. If both laser rangefinders detect the same low range from obstacles, UAV will climb to fly over them. These two possibilities of flight control are fundamentals of autonomous flight of unmanned aerial vehicle. Moreover, the simultaneous combination of turn and climbing is available using the proposed obstacle avoidance strategy, what increases vehicle agility. It is because of the obvious fact that climbing can highly reduce the ground projection of turn radius. The equipment used in the experiment can be easily built into micro unmanned aerial vehicle with a wing span about 1 meter, and it is truly impressive success of the research.

\section{REFERENCES}

1. Andert F., Adolf F., Goormann L., Dittrich J. (2010), Autonomous Vision-Based Helicopter Flights Through Obstacle Gates, Journal of Intelligent \& Robotic Systems, Vol. 57, Issue 1-4, 259-280.

2. Beyeler A., Zufferey J.C., Floreano D. (2009), Vision based control of near-obstacle flight, Autonoums Robots, Vol. 27, № 3, 201-219.

3. Campoy P., Correa J. F., Mondragón I., Martínez C., Olivares M., Mejías L., Artieda J., Valavanis K., Oh P., Pieg L. (2009), Computer Vision Onboard UAVs for Civilian Tasks, Unmanned Aircraft Systems, Springer Netherlands, Vol. 54, Issue 1-3, 105-135.

4. Griffiths S., Saunders J., Curtis A., Barber B., McLain T., Beard R. (2007), Obstacle and Terrain Avoidance for Miniature Aerial Vehicles, Advances in Unmanned Aerial Vehicles, State of the Art and the Road to Autonomy, Spinger, 2007, 213-244.

5. Hrabar S. E., Sukhatme G. S. (2006), Optimum camera angle for optic flow-based centering response, IEEE/RSJ International Conference on Intelligent Robots and Systems (IROS), 3922 - 3927.

6. Hrabar S. E., Sukhatme G. S. (2009), Vision-Based Navigation Through Urban Canyons, Journal of Field Robotics, Vo.26, Issue 5, $431-452$.

7. Kownacki C. (2009), Guidance and obstacle avoidance of MAV in uncertain urban environment, European Micro Aerial Vehicle Conference and Flight Competition 2009: EMAV'2009 Delft, CDROM.

8. Kownacki C. (2010), Algorithm development for flight control of micro aircraft in uncertain urban environment, Acta Mechanica et Automatica, Vol. 4, No. 3, 87-93, (in Polish).

9. Kownacki C. (2011), Obstacle avoidance strategy for Micro Aerial Vehicle, Advances in aerospace guidance, navigation and control, Berlin: Springer-Verlag, 117-135.

10. Todorovic S., Nechyba M.C. (2004), A Vision System for Intelligent Mission Profiles, IEEE Transactions On Vehicular Technology, Vol. 53, №. 6, 1713-1725.

The research was funded by funds of the Polish Ministry of Science and Higher Education for science in 2008-2010 as a developmental project No O R00 0059 06. Very big thanks to Jaroslaw Hajduk for his invaluable help in flights and vehicle assembling. 